\title{
VOM PROFESSOR ZUM STADTSCHREIBER: M. JACOBUS CODICILLUS († 1576) UND SEINE BIBLIOTHEK
}

\author{
JIŘÍ PEŠEK
}

\author{
FROM PROFESSORSHIP TO TOWN SCRIBE: M. JACOBUS CODICILLUS († 1576) \\ AND HIS LIBRARY
}

The study contemplates possibilities for a closer understanding of the society of university masters and seeks to approach them through inventories of their libraries. As an example, it gives the inventory of the estate of Master Jakub Codicillus ( $†$ 1576), which provides a record of some 216 handwritten and printed books. The study presents the contents of this library.

Keywords: Master Jakub Codicillus - University of Prague - Prague - sixteenth century - libraries - inventory

DOI: $10.14712 / 23365730.2020 .24$

Zu Beginn sei die Schlüsselfrage gestellt: warum interessiert man sich eigentlich für Privatbibliotheken der frühneuzeitlichen Professoren, besonders dann, wenn sie keine international berühmten Persönlichkeiten waren? Die Antwort könnte lauten, dass einfach alles, was die bis heute nur wenig durchforschte Geschichte der europäischen Universitäten näher zu verstehen hilft, für uns wichtig sein sollte. Ganz konkret aber geht es uns im Prager Fall darum, die geistige Atmosphäre und konfessionelle Orientierung der protestantischen Universität der zweiten Hälfte des 16. Jahrhunderts besser zu beleuchten und vielleicht auch etwas über ihr intellektuelles Niveau sagen zu können. ${ }^{1}$

Die Prager utraquistische, also nichtkatholische und in dem untersuchten Zeitraum zwar nicht offen lutherische, aber dem Wittenberger und Leipziger Luthertum kulturell und konfessionell nahe stehende Universität oder, mit einem zeitgenössischen Begriff bezeichnet: „Akademie“, hat schon traditionell einen eher schlechten Ruf in der Geschichtsliteratur:2 Bis heute werden ohne jede Kritik die Ansichten des hochverdienten Forschers des späten 19., frühen 20. Jahrhunderts Zikmund Winter wiederholt, der mit einer fast unübertroffenen Quellenkompetenz und einer liebenswürdigen Zuneigung fürs Detail, sonst aber mit einer nur für bestimmte Fragen relevanten Mosaiksteinchen-Methode seine Thesen über Städte, Schulen und natürlich auch über die ,hussitische“ Universität der Reformationszeit

1 Zur Geschichte der Prager Universität im 16. Jahrhundert allgemein vgl.: Michal Svatoš, The Utraquist University 1419-1622, in: František Kavka - Josef Petráň (eds.), A History of Charles University, I, Prague 2001, S. $195-197$.

2 Ein Versuch, diese Problematik etwas optimistischer zu präsentieren, in: Jiří PeŠEK, Humanism and Teaching at Prague University in the Pre-White Mountain Period, ibidem, S. 203-217. 
formulierte. ${ }^{3}$ Er tat dies übrigens als katholischer Historiker immer mit bestimmten Vorurteilen und ohne einen - damals übrigens kaum möglichen - internationalen Vergleich.

Die Universitätsforschung der jüngsten Dekaden zeigte allerdings, dass die erhaltenen Quellen akademischer Provenienz die reale kulturelle Situation nur unzureichend beleuchten. Die Lage der mitteleuropäischen Universitäten in der Zeit der Wirren der europäischen Reformation kam praktisch nirgendwo dem hohen, abstrakt akademischen Ideal nahe. Und vor allem: für zusammenfassende Urteile und Interpretationen benötigen wir eine Menge an konkreten analytischen Studien. ${ }^{4}$ Wie soll man aber die intellektuelle Situation einer Universität konkret erforschen? Dafür bietet sich eine Analyse der publizierten oder im Manuskript erhaltenen Schriften einzelner Professoren an bzw. die Untersuchung der Thesen, welche bei den Bakalaureatsprüfungen oder Magisterverfahren disputiert wurden. ${ }^{5}$ Eher selten erhalten sind schriftlich verfasste Unterlagen oder Notizen aus den - noch im 16. Jahrhundert ab und da gehaltenen - Disputationen oder öffentlichen Reden. Memoriale Eintragungen im Liber decanorum facultatis philosophicae bzw. in den Acta rectorum Universitatis Pragensis oder die für die breite Öffentlichkeit bestimmten Texte universitärer Intimationen sind wertvoll, aber meistens zu kurz, um auf ihrer Basis größere Interpretationen zu wagen. ${ }^{6}$

Die Aufmerksamkeit, welche die Forschung den mit der frühneuzeitlichen Universität verbundenen Bibliotheken widmet, ist also durchaus berechtigt und knüpft daneben an eine lange Tradition der Untersuchung mittelalterlicher Codices im akademischen Milieu an. ${ }^{7}$ Die Stellung und Rolle des Buches hat sich aber einhundert Jahre nach der Erfindung des Buchdrucks, nach dem Etablieren eines sehr flexiblen, praktisch gesamteuropäischen Buchmarktes und nach der Vermassung des gedruckten Buches in der kommerziellen Konsequenz der lutherischen Reformation grundsätzlich verändert. ${ }^{8}$ Die alten handgeschriebenen Bestände der Kollegienbibliotheken verloren an Wert und Attraktivität. Die Flut der relativ billigen, nach Bestellung schnell erreichbaren gedruckten Bücher zu jedem Thema

3 Zikmund WINTER, Děje vysokých škol pražských od secessí cizich národů po dobu bitvy bělohorské (1409-1622) [Geschichte der Prager Hochschulen vom Auszug der fremden Universitätsnationen bis zur Schlacht am Weißen Berg (1409-1622)], Praha 1897; Ders., O životě na vysokých školách pražských knihy dvoje. Kulturní obraz XV. a XVI. století [Zwei Bücher über das Leben an den Prager Hochschulen. Ein Kulturgemälde des XV. und XVI. Jahrhunderts], Praha 1899.

4 Als Inspiration eignen sich für uns vor allem zahlreiche Studien über die Wiener Universität des 16. Jahrhunderts. Vergleiche: Kurt MüHlBerger, Die Gemeinde der Lehrer und Schüler - Alma Mater Rudolphina, in: Peter Csendes - Ferdinand Opll (Hgg.), Wien, Geschichte einer Stadt, I, Von den Anfängen bis zur Ersten Türkenbelagerung (1529), Wien - Köln - Weimar 2001, s. 319-410; DERs., Zu den Krisen der Universität Wien im Zeitalter der konfessionellen Auseinandersetzungen, in: Bericht über den achtzehnten österreichischen Historikertag in Linz vom 24. bis 29. September 1990 (=Veröffentlichungen des Verbandes Österreichischer Geschichtsvereine 27), Wien 1991, S. 269-277; Kurt Mühlberger - Meta Niederkorn Bruck (Hgg.), Die Universität Wien im Konzert europäischer Bildungszentren 14.-16. Jahrhundert, Wien - München 2010.

5 Jiří PeŠEx, Political Culture of the Burghers in Bohemia and Central Europe during the Pre-White Mountain Period, in: Halina Manikowska - Jaroslav Pánek - Martin Holý (eds.), Political Culture in Central Europe (10th - 20th Century), I, Middle Ages and Early Modern Era, Prague 2005, pp. 203-214.

6 František Šmahel - Gabriel Silagi (edd.), Statuta et Acta rectorum Universitatis Carolinae Pragensis 1360-1614, Praha 2018.

7 Siehe den Beitrag von Ivan HLAváČEK in diesem Band.

8 Prag lag im Schnittpunkt der Einflusssphären vor allem der großen internationalen Buchhandelsfirmen in Nürnberg (Anton Koberger) und Leipzig (Peter Drach). Später spielten die Frankfurter und seit den neunziger Jahren des 16. Jahrhunderts auch die Leipziger Buchmessen eine entscheidende Rolle. Vgl. schlüssig: Reinhard Wittmann, Geschichte des deutschen Buchhandels, München 1991. 
ermöglichte es den Professoren damals, große Privatbibliotheken mit persönlichen thematischen Schwerpunkten aufzubauen. ${ }^{9}$

Es ist also gut nachvollziehbar, dass diese - das intellektuelle Profil ihrer Inhaber mindestens in gewissem Maße widerspiegelnden - Büchersammlungen zum Forschungsthema geworden sind. Die Problematik hat aber doch auch bestimmte Tücken: Erstens handelt es sich um die Quellenfrage. Man muss damit rechnen, dass die meisten Bibliotheken im bürgerlichen Milieu - und in diese Schicht gehörten auch die Professoren der Prager Universität - den Tod ihrer Besitzer höchstens um eine Generation überlebten. Dann wurden sie verkauft und nur selten den Bibliotheken der Kollegienhäuser einverleibt. Die Tatsache, dass eine Mehrheit der Professoren nach einigen Jahren ihre mit dem Zölibat verbundene Stelle durch eine günstige Heirat oder durch den Wechsel auf prestigevolle Posten in der Stadtverwaltung aufgab, brachte es mit sich, dass sich auch ihre Bibliotheken dem akademischen Milieu entzogen.

Spätestens nach den dramatischen Ereignissen nach dem Scheitern des böhmischen Ständeaufstandes von 1620 wurden die Prager Bibliotheken teilweise vernichtet oder entfernt, teilweise grundsätzlich durchgemischt. Intakte Bibliothekseinheiten aus dem akademischen Milieu sind also nicht erhalten. Nur als Glücksfall, als Gabe an die Stadtschule seiner Geburtsstadt, überlebte die Bibliothek eines - sagen wir etwas schwierigen - Prager Professors aus dem untersuchten Zeitraum: Gallus Gelastus aus Vodňany. Diese Bücherkollektion wurde schon im gedruckten Katalog an die Öffentlichkeit vermittelt. ${ }^{10}$

Mehr Spuren der damaligen Professorenbibliotheken entdecken wir in ihren Nachlassinventaren, die in die Prager Stadtbücher eingetragen wurden. ${ }^{11}$ Diese Quellen betreffen jene Professoren, welche ihre etwas karge akademische Karriere durch bessere Möglichkeiten im städtischen Bereich ersetzt hatten. Falls nach ihrem Ableben komplizierte Erbschaftsumstände festgestellt wurden oder beispielsweise die väterliche Bibliothek dem Sohn für seine Studien erhalten bleiben sollte, wurde sie amtlich, also durch eine Ratsherrenkommission verzeichnet. ${ }^{12}$ Das Problem besteht in der Tatsache, dass diese Kommissionen vor allem Wertgegenstände und Eigentum allgemein auflisten mussten. Die meisten Bücher waren in der Kokurrenz mit Immobilien, Schuldbriefen, Bargeld oder Goldketten einfach zu billig. Man verzeichnete sie also entweder nur summarisch oder hielt vor allem kostspielige, gut gebundene Bücher großer Formate fest, während kleine oder nicht ordentlich gebundene also billige oder finanziell uninteressante - Bände erst gar nicht erwähnt wurden.

9 Zu dieser Thematik gibt es bereits eine Reihe von Büchern und Studien. Vgl. zur böhmischen Situation: Jiří PEŠEK, Prager Leser der rudolfinischen Zeit, in: Hans B. Harder - Hans Rothe (Hgg.), Später Humanismus in der Krone Böhmen 1570-1620, Dresden 1998, S. 217-224; Olga FEJTOvÁ - Jiří PEŠEK, Reflexion der böhmischen und europäischen konfessionellen Tradition in frühneuzeitlichen böhmischen bürgerlichen Bibliotheken, Bohemia 48, 2008, S. 29-53.

10 Karel Platzer, Knihovna M. Havla Gelasta Vodňanského [Die Bibliothek des Magisters Gallus Gelastus Vodnianus], Vodňany 1987.

$11 \mathrm{Zu}$ dieser Quelle vergleiche: Jiří PEŠEK, Pražské knihy kšaftů a inventářru pozůstalostí-přispěvek k jejich struktuře a vývoji v době predbělohorské [Die Prager Bücher der Testamente und Nachlassinventare - ein Beitrag zu ihrer Struktur und Entwicklung in der Zeit vor der Schlacht am Weißen Berg], Pražský sborník historický (weiter PSH) 15, 1982, S. 63-92.

12 Jiř́ PEŠEK, Knihy a knihovny v kšaftech a inventář́ch pozůstalostí Nového Města pražského v letech 1576-1620 [Bücher und Bibliotheken in den Testamenten und Nachlassinventaren der Prager Neustadt in den Jahren 1576-1620], Folia Historica Bohemica 2, 1980, S. 247-282. 
Diesen Problemen begegnen wir im Falle der Bibliotheken z. B. des Prager Kanzlers und ehemaligen Professors Paul Christian von Koldin (er starb 1589) oder des etwas jüngeren ehemaligen Professors Johannes Kanha von Weleslavin. Dieser frisch eingesetzte Neustädter Ratsherr, der 1599 einer Pestepidemie zum Opfer fiel, war Inhaber der größten Privatbibliothek (das Nachlassinventar verzeichnet 414 Exemplare, von denen allerdings nur ein einziges, das prächtige Theatrum orbis Ortelii, namentlich erfasst ist), die im Prag des langen 16. Jahrhunderts ermittelt werden konnte. ${ }^{13}$

Auch wenn Verzeichnisse von Privatbibliotheken erhalten sind und wir mehr oder weniger entschlüsseln können, welche konkreten Bücher hinter den durch die Stadtschreiber aufgelisteten Kurztiteln stecken, stellt sich die Frage nach der Aussagekraft dieser Inventare: Haben die Buchbesitzer ihre Exemplare auch wirklich gelesen? Jeder Editor von wissenschaftlichen Zeitschriften stellt sich auch heute - in einer Zeit, da es offenbar nichts Wichtigeres als den Zitationsindex gibt - diese Frage sehr oft bei den eingereichten Texten, denn nicht selten entsprechen diese nur teilweise dem Inhalt der im Apparat erwähnten Werke und umgekehrt fehlen Hinweise gerade auf jene Titel, welche für die untersuchte Problematik grundlegend sind. War das im 16. Jahrhundert anders? Wir rechnen also zumindest damit, dass die Buchbesitzer ihre Bücher mit der Absicht bestellt und gekauft hatten, diese Texte für einen bestimmten Zweck zu gebrauchen. Wir können aber auch damit rechnen, dass die Häufigkeit, mit welcher der Buchbesitzer zu Lebzeiten zu den einzelnen Bänden unter den aufgelisteten Büchern griff, ganz unterschiedlich war.

Kommen wir nun zum eigentlichen Gegenstand unserer Untersuchung: zu Professor Jacobus Codicillus. ${ }^{14}$ Dieser Sohn eines Tuchhändlers namens Jan Codex alias Bradáč aus der in ländlicher Umgebung im südlichen Mittelböhmen gelegenen Stadt Sedlčany wurde um 1530 geboren. Er absolvierte sein Bakkalaureat $15488^{15}$ mit einer Disputation der These Ob man wegen der Undankbarkeit des Volkes Studien vernachlässigen soll und erwarb 1550 seinen Magistergrad nach Verteidigung der These Ob die Schulen das in der Gemeinde wertvollste Kleinod sind. ${ }^{16}$ Schon zuvor, aber auch kurz danach wirkte er als Schulrektor in Chrudim, erwarb für sich und seinen jüngeren Bruder Peter das Nobilitätsprädikat „,von Tulechov“ und wurde schon 1551 an die Universität berufen. ${ }^{17}$ Dort sind seine Vorlesungen über Aristotelische Physik belegt. ${ }^{18}$ In dem stabilen, dem Curriculum entsprechenden System lehrte er aber auch über andere Werke des Aristoteles. ${ }^{19}$ Alsbald

13 Jiří Pešek, Pavel Kristián z Koldina - život a kariéra [Paul Christian von Koldin - Leben und Karriere], in: Karel Malý - Jiří Šouša (edd.), Městské právo ve střední Evropě, Praha 2013, S. 25-62; TÝž, Mistr Jan Kaňha z Veleslavína - obyčejný život pražského profesora a měštana doby rudolfínské [Magister Johannes Kaňha von Veleslavin - das gewöhnliche Leben eines Prager Professors und Bürgers der rudolphinischen Zeit], AUC-HUCP 47/1-2, 2007, S. 161-170.

14 Vgl. das biographische Lexikon des böhmischen Humanismus: Josef HEJNIC - Jan MartíneK, Rukovět’ humanistického básnictví v Čechách a na Moravě. Enchiridion renatae poesis [Vademecum der humanistischen Dichtkunst in Böhmen und Mähren. Enchiridion renatae poesis], I, A-C, Praha 1966, S. 387-388.

15 Am 13. Juni 1548 im Liber decanorum facultatis philosophicae Universitatis Pragensis ab anno Christi 1367 usque ad annum 1585, Pragae 1832, S. 346 unter dem Namen Jacobus Sedlcžanus eingetragen.

16 Liber decanorum, S. 351, wo er bereits unter dem Namen Jacobus Codicillus Sedlcžanus a Tulechowa geführt wird.

17 Unter dem Dekan Mathias Bydzowinus ab Aventino wurde er einer der beiden Professoren, die ,pro pecuniis in fisco" zuständig waren. Vgl. Liber decanorum, S. 356.

18 J. HeJNIC - J. MARTínEK, Rukovět', S. 387.

19 Noch nach Codicillus' Tod befand sich ein Werk des Aristoteles in seiner Bibliothek: Politeia et Oeconomia Aristotelis. Dieses Buch stand für die Luthersche Ethik im zeitgenössischen Kontext. Vgl. Risto SAARINEN, 
wurde er auch als erfolgreicher Arzt bekannt. Schon von 1555 bis 1557 diente er seiner Fakultät zweimal als Dekan. ${ }^{20}$ Bald danach aber endete seine kurze akademische Laufbahn, als er 1557 eine bürgerliche Tochter aus der Prager Neutstadt heiratete. Doch noch bis 1559 hielt er Vorlesungen, dann wurde er Stadtschreiber und Vizekanzler der Prager Neustadt. Dies war eine sehr prominente Stellung im Rahmen der Prager bürgerlichen Elite.

Jacobus Codicillus blieb in guten Beziehungen zum Kreis der Professoren und den mit der Universität verbundenen böhmischen Humanisten. $\mathrm{Zu}$ dieser freundschaftlichen und in Prag wie auch im ganzen Königreich einflussreichen Gruppe gehörten die wichtigsten Persönlichkeiten des an Wittenberg orientierten Humanismus: genannt seien Codicillus' Universitätslehrer Jan Hortensius, der Gräzist Matthias Collinus, die Hauptperson des Prager Humanismus jener Zeit, ferner Sebastian Aerichalcus oder der gelehrte Jurist Jakub Srnovec von Warwazov. Codicillus' Verbindung zur Universität war umso stärker, als sein jüngerer Bruder, Peter Codicillus, nach Studien in Wittenberg und einer üblichen Dienstzeit an den böhmischen Stadtschulen 1561 eine Professur in Prag übernommen hatte und zu einem der profiliertesten und produktivsten Prager akademischen Humanisten wurde. ${ }^{21}$

Nach seinem Ausscheiden aus dem akademischen Milieu war Jacobus Codicillus nicht mehr literarisch tätig. Seine Position im Rathaus, seine medizinische Praxis, Familie und auch seine erfolgreiche wirtschaftliche Betätigung ließen offensichtlich wenig Zeit dafür übrig. Es wird tradiert, dass er ein begeisterter und erfolgreicher Sänger im Literatenchor an der Pfarrkirche des hl. Stephanus in der oberen Prager Neustadt gewesen sei; auch habe er ein vierbändiges Gesangbuch verfasst. ${ }^{22}$ Diese Leistungen bezeugen sein Prestige in der Gemeinde, sagen allerdings nur wenig über seine wirkliche musikalische Begabung und Aktivität aus. Es ist auch nicht ausgeschlossen, dass diese Berichte eher seinen jüngeren Bruder Peter betreffen. ${ }^{23}$

Wir wissen also sehr wenig über Codicillus' kulturelle Aktivitäten nach seinem Wechsel in städtische Dienste. Seine für den untersuchten Zeitraum außerordentlich große Bibliothek wuchs aber offensichtlich auch in dieser Zeit und spiegelt seine breiten und vielleicht zunehmend auch politischen und konfessionellen Interessen wider. Als Codicillus nach einer dreijährigen schweren Krankheit am 21. September 1576 starb und in der Neustädter Stephanskirche bestattet wurde, musste man - obwohl seine Frau noch lebte - wegen der

Ethics in Luther's Theology: The Three Orders, in: Jill Kraye - Risto Saarinen (eds.), Moral Philosophy on the Treshold of Modernity, Dordrecht 2005, S. 195-213, hier S. 202. Im Zusammenhang mit Aristoteles gab es noch ein Buch in Codicillus' Bibliothek, nämlich Notizen zu Aristoteles, eines der frühen Werke von Pièrre DE LA Ramée, Petri Rami animadversionum Aristotelicarum. Hierbei handelt es sich um eines der ersten, schon 1543 in Paris bei Iacobus Bogardus unter dem Titel Aristotelicae Animadversiones gedruckten Werke dieses jungen, noch katholischen Philosophen. Das Buch erschien gleichzeitig mit zwei weiteren Werken von Ramus über Dialektik: Dialecticae institutiones und Dialecticae partiones, und bezeugt das große Interesse sowohl des jungen Franzosen als auch des ebenfalls jungen Prager Professors für die Dialektik der Klassiker. Vgl. auch: Petrus Ramus, Dialecticae Institutiones; Aristotelicae Animadversiones. Facsimile-Neudruck der Ausgabe Paris 1543 mit einer Einleitung von Wilhelm Risse, Stuttgart - Bad Canstatt 1964.

20 Liber decanorum, S. 361 und 363.

21 J. Hejnic - J. MaRTíneK, Rukovět', S. 389-402.

22 Bohumír Jan Dlabač, Allgemeines historisches Künstler-Lexikon für Böhmen und zum Theil auch für Mähren und Schlesien, Prag 1815, I, Spalte 286. Das behaupten auch: J. HeJNIC - J. MARTíneK, Rukovět', I, S. 388.

23 Vgl. Karel KonráD, Dějiny posvátného zpěvu staročeského od 15. věku do zrušeni literátských bratrstev [Geschichte des altböhmischen Sakralgesangs vom 15. Jahrhundert bis zur Aufhebung der Literatenbruderschaften], I, Praha 1893, S. 124. 
Notwendigkeit, für seine vier, teilweise kleinen Kinder einen Vormund zu bestimmen, seinen Nachlass inventarisieren. ${ }^{24}$

Leider fehlen die beiden ersten Folien des Stadtbuches, in dem Codicillus' Nachlass als erster Eintrag aufgenommen wurde: Vom gesamten Inventar seines Eigentums ist uns also nur die ganz und gar ungewohnt ausführliche Auflistung seiner großen Bibliothek erhalten geblieben. Offensichtlich hatte die Tatsache, dass Codicillus' ältester Sohn Johannes damals schon in Leipzig Medizin studierte, ${ }^{25}$ die vom Rathaus eingesetzte Inventarisierungskommission dazu bewogen, die Bibliothek bzw. ihre großformatigen Bücher namentlich zu verzeichnen. Dank dieser Tatsache liegt uns eine Liste vor, welche alle größeren, also in folio bis quarto gedruckten Bücher anführt, die sich in dem großen Zimmer seines Hauses in dem reichen Stephanus-Viertel befanden. ${ }^{26}$

Diese Bücherliste von 216 Titeln war in zwei Teile gegliedert: In dem ersten, allgemeinen Teil finden wir vor allem großformatige Bände religiöser, vor allem lutherischer, aber auch kalvinistischer Literatur, und zwar in einem breiten Spektrum. Im Zentrum dieser Büchersammlung standen ganz natürlich mehrere Werke von Martin Luther, Philipp Melanchthon und Desiderius Erasmus, aber auch von Johann Spangenberg. ${ }^{27}$

Das Interesse an anderen, vor allem schweizerischen religiösen Richtungen der Reformation belegen die Werke von Heinrich Bullinger oder gar Johannes Calvinus. Seine Institutio Christiane Religionis, dieser „Kathechismus für Gebildete“, wie der Verfasser sein Werk im Geleitwort des Buches charakterisiert, gehört zu den in den Prager Bibliotheken wahrlich seltenen Büchern. ${ }^{28}$ Man kann vermuten, dass sich Codicillus diese Bücher im Zusammenhang mit den Vorbereitungen auf die Debatten über die Böhmische Konfession von 1575 beschafft hatte. Als Beweis für diese These kann man die im Bibliotheksinventar vorhandene Apologia Verae Doctrinae fratrum Boemorum anführen..$^{29}$ Codicillus war zwar schon seit 1574 krank, Überlegungen aber über eine gemeinsame Konfession, welche neben der - übrigens lutheranisierten - utraquistischen Kirche auch die deutschen Lutheraner und die ihnen in dieser Zeit nahestehende Brüderunität umfassen würde, wurden schon seit dem Landtagsbeschluss von 1567 über die Aufhebung der für die aktuelle Situation zu

24 Vgl. Archiv der Hauptstadt Prag, Handschriftensammlung, Handschrift Nr. 1211 (Buch der Nachlassinventare der Prager Neustadt - Stephansviertel 1577-1605), Fol. 1 bis 3. „Inventář pozi̊stalosti po M. Jakubu Kodicillovi z Tulechova“ [Nachlassinventar des Magisters Jacobus Codicillus von Tulechov]. Die Witwe Kateřina starb 1582. Vgl. ihr Nachlassinventar in demselben Stadtbuch Nr. 1211, Fol. $36 \mathrm{~b}$ bis 41 a.

25 Johannes Codicillus promovierte 1579 in Tübingen, ist dann aber noch nach Straßburg gegangen. Er starb kurz nach seiner Rückkehr in Prag im Jahre 1585. Seine - vom Vater geerbte - Bibliothek ist an Peter Codicillus weitergegangen. Vgl. J. HeJNIC - J. MARTíNeK, Rukovět', S. 388-390.

26 Eine analytische Edition dieses Verzeichnisses der Codicillschen Bibliothek befindet sich erst in Vorbereitung.

27 Olga FejtovÁ - Jiří PešeK, Erasmus, Luther und Melanchthon in den Privatbibliotheken der böhmischen Bürger um 1600, Colloquia. Journal of Central European History, V-VII, 1998-2000, S. 66-93; DieselBen, Martin Luther in den Bibliotheken böhmischer Bürger um 1600: Zur Rezeption und Wirkung von Luthers Lehre, Bohemia 37, 1996, S. 319-340; Dieselben, Postila Johanna Spangenberga v měštanských knihovnách raného novověku [Postille des Johann Spangenberg in den bürgerlichen Bibliotheken der Frühen Neuzeit], Documenta Pragensia 23, 2004, S. 101-122.

28 Vgl. Institutio Christianae Religionis Ioannis Caluini, Handschrift Nr. 1211, Fol. 1a. Die erste - lateinische Ausgabe des Werkes erschien 1536 in Basel.

29 Apologia Verae Doctrinae fratrum Boemorum, Handschrift Nr. 1211, Fol. 1b. Es handelt sich wahrscheinlich um den Druck eines Werkes von Jan Augusta (die deutsche Ausgabe der Apologia erschien schon 1532 in Augsburg): Apologia verae Doctrinae eorum vulgo appellantur Waldensium et Picardorum in Bohemia, Wittenberg 1538. Die Vorrede zu dieser Ausgabe stammt wahrscheinlich von Philipp Melanchthon. 
eng geschnittenen hussitischen Kompaktaten von 1436 und des Kuttenberger Religionsfriedens von 1485 angestellt. ${ }^{30}$

Codicillus' breitgefächertes Interesse beweisen auch solche Bücher wie die Historia Hussitarum, also eine von Johannes Dobeneck alias Cochläus, einem katholischen Mainzer Prälaten und Gegner Luthers, im Jahre 1549 publizierte Polemik über die Hussiten und ihre lutherischen Nachfolger. ${ }^{31}$ Gleich daneben, also noch unter den theologischen Büchern stand das Chronicon Eusebii de Caesarea, eine ursprünglich griechische Kompilation der Weltgeschichte von Abraham bis Kaiser Constantinus. Dieses Buch wurde seit 1475 wiederholt gedruckt. ${ }^{32}$

Die andere Hälfte der Bibliothek trug im Inventar die Überschrift Philosophici libri. Diese fast einhundert Bücher zunächst in folio, dann in quarto, waren mit der Medizin und mehr oder weniger mit dem akademischen Milieu und dem Humanismus verbunden. Die vierzehn medizinischen Bücher bieten uns ein Kompromissbild: Auf der einen Seite stand die traditionelle Medizin des Mittelalters mit den Schriften von Hippokrates oder Galen und mit verschiedenen Kommentaren dazu, auf der anderen Seite finden wir dann die berühmte Anatomia von Andreas Vesalius, Werke von Leonhard Fuchs oder Antonius Musas De medicamentis tam simplicibus, also die aktuellen grundlegenden Werke der Renaissancemedizin. ${ }^{33}$

Unter den nicht-medizinischen Titeln offenbart sich uns dann ein Panorama des Humanismus, das von der zahlreichen klassischen griechischen und römischen Literatur bis zur antiken Philosophie und den ganz neuen Kommentaren und Nachschlagewerken reicht, welche dieses intellektuelle Erbe in der Renaissance aktuell interpretierten. Wieder spielen hier vor allem Philipp Melanchthon und der schon erwähnte Petrus Ramus eine bedeutende Rolle. Man findet hier aber auch juristische, politische Titel und einige Chroniken. ${ }^{34}$

30 Zur allgemeinen Situation vgl. Zdeněk V. DAVID, Nalezení střední cesty. Liberální výzva utrakvistů Řimu a Lutherovi [Einen Mittelweg finden. Eine liberale Aufforderung der Utraquisten an Rom und Luther], Praha 2012, S. 291-336.

31 Handschrift Nr. 1211, Fol. 2b: Historia Hussitarum libri duodecim per Joannem Cochlaeum... Cum Philippica septima Jo. Cochlaei, de Publica Caroli V imperatoris ordinatione quae vulgo Interim dicitur... Ejusdem... aedentur deinceps ... ab eodem Cochlaeo Commentaria de actis \& scriptis Martini Lutheri, Mainz Franz Behem 1549.

32 Im Kontext der untersuchten Bibliothek erscheinen die Baseler Ausgaben aus den Jahren 1529 oder 1536 wahrscheinlicher als die zahlreichen Pariser Editionen. Die Fassung der Aufzeichnung erinnert aber eher an die venezianische Inkunabel: Chronicon Eusebii, a sancto Hieronymo latine versum et ab eo, Prospero Britannico et Matthaeo Palmerio continuatum, editum cura C. L. Johannis Hippodami, Venedig 1483.

33 Vgl. Jiří PEŠEK, Zdravotnická literatura v pražských měštanských knihovnách přelomu 16. a 17. století [Die Medizinliteratur in den Bibliotheken der Prager Stadtbürger an der Wende vom 16. zum 17. Jahrhundert], Documenta Pragensia 7, 1988, S. 236-252. Zur Bibliothek von Codicillus vgl. S. 237f. und S. 242.

34 Nur als Beispiel: Welche Kodifikation des böhmischen Städterechts sich unter der Bezeichnung Prava Miestska stara in Codicillus' Bibliothek verbarg, ist schwierig zu sagen. Dagegen war das Vocabularius Utriusque Iuris offensichtlich das Vocabularius utriusque iuris. Item modus legendi abbreviaturas, Lugdunum 1559. Unter Ioannes Ssleidanis de statu Religionis finden wir den zeitgeschichtlich-politischen Kommentar von Ioanni Steidani De Statu Religionis Et Reipublicae Carolo V. Caesare Commentarii, Genf, Druckerei Badius 1559. Der Liber Chronicarum ab initio mundi wird am ehesten das monumentale, bei Anton Koberger gedruckte Werk von Hartmann Schedel, Liber Chronicarum. Registrum huius operis libri cronicarum cum figuris et ymagibus ab initio mundi, Nürnberg 1493 gewesen sein. Vgl. dazu Jiř́ PEšEK, From veduta to photography of the city: The staging of the city and its history, in: Olga Fejtová - Václav Ledvinka - Martina Maříková - Jiří Pešek (edd.), Od veduty k fotografii. Inscenování města v jeho historii, Praha 2017, S. 695708, hier S. 696f. Dagegen kann man leider die in der handschriftlichen Form erhaltene lateinische Chronik von Pulkava von Radenin, welche in J. HeJniC - J. MartíneK, Rukovět', I, S. 387 als Leihgabe an Procopius 
Wie soll man nun diese umfangreiche Bibliothek als Ganzes charakterisieren?

Der allgemeine Teil der Büchersammlung zeigt uns ein großes Interesse ihres Besitzers an religiöser Literatur. So etwas war in dieser durch erregte konfessionelle Diskussionen geprägten Zeit in Prag durchaus üblich. Die Büchersammlung von Jacobus Codicillus zeigt aber auch seine unüblich breite Orientierung in der aktuellen - so zerstrittenen und zugleich verflochtenen - Reformationsliteratur des Reichsgebietes und der Schweiz. Es überrascht allerdings, dass in dieser Prager Sammlung die böhmische Buchproduktion zur konfessionellen Problematik fast völlig fehlt.

Bei Codicillus findet man auch einige Exemplare der wichtigsten zeitgenössischen juristischen Literatur, ${ }^{35}$ was bei einem gelehrten Stadtfunktionär kaum überrascht. Die Abteilung der philosophischen Literatur spiegelt dann sowohl die Lehrprogramme der städtischen Lateinschulen als auch der Artistenfakultät wider. Auch in dieser thematischen Gruppe finden wir zahlreiche Beweise für das besondere Interesse, das Codicillus an aktuellen Tendenzen der Philosophie, der Historiographie und natürlich auch der Medizin hatte.

Worüber wir hingegen von dieser großartigen Bibliothek kaum etwas sagen können, ist ihre Positionierung im Kontext der Prager Büchersammlungen, welche von vergleichbaren, akademisch geprägten und mit der Prager Universität weiterhin in Kontakt stehenden Persönlichkeiten in jener Zeit möglicherweise ebenfalls angelegt wurden. ${ }^{36}$ Kein Zweifel besteht, dass die Bibliothek von Codicillus nicht nur groß, sondern auch inhaltlich ausgezeichnet war. Doch wird man weder behaupten können, dass diese, einen offenen Intellekt mit breiten Horizonten ihres Besitzers bezeugende Bibliothek für das Prager städtisch-akademische Elitenmilieu typisch war, noch dass sie eine Ausnahme darstellte. Man kann lediglich aus der Perspektive eines Kulturhistorikers behaupten, dass sie eine der inhaltlich interessantesten Büchersammlungen ist, denen man in der langjährigen Forschung über die Prager Bibliotheken des halben Jahrhunderts vor Beginn des Dreißigjährigen Krieges bislang begegnen konnte.

(Deutsche Fassung: Autor; sprachliche Korrektur: Wolf B. Oerter)

Lupacius erwähnt wird, in der Bücherliste nicht finden, weil dort wahrscheinlich nur großformatige Bücher verzeichnet waren.

35 Vgl. Olga FejtovÁ - Jiří PeŠEK, ,,... a obzvláště jedna truhla kněh právních. “ Právnická literatura v městech pražských raného novověku (na přikladu Nového Města pražského) [,... und insbesondere eine Truhe mit juristischen Büchern." Die Rechtsliteratur in den Prager Städten der Frühen Neuzeit], PSH 42, 2014, S. 43-87.

36 Grenzüberschreitende Vergleiche innerhalb Mitteleuropas sind auch nur wenig hilfreich. Die beeindruckende, aktuelle Bearbeitung des Katalogs von Hieronymus Beck durch Lenka Veselá führt uns in ein doch höheres höfisches Milieu. Vgl.: Lenka Veselá, Ritter und Intellektueller - Hieronymus Beck von Leopoldsdorf (1525-1596) und seine Bibliothek, Frankfurt am Main - Bern - Wien 2017. Vergleiche mit den nach ganz anderen Prinzipien aufgebauten Bibliotheken der Aristokratie helfen dann noch weniger. Vgl. exemplarisch: Lenka Veselá, Knihy na dvoře Rožmberků [Bücher am Hof der Rosenberger], Praha 2005, oder Ivo PuRš Hedvika KuchaŘovÁ (edd.), Knihovna arcivévody Ferdinanda II. Tyrolského [Die Bibliothek Erzherzog Ferdinands II. von Tirol], Praha 2015. 
JIŘÍ PEŠEK

\section{OD PROFESURY K PROFESI MĚSTSKÉHO PÍSAŘE: \\ M. JACOBUS CODICILLUS († 1576) A JEHO KNIHOVNA}

\section{RESUMÉ}

Studie se zamýšlí nad možnostmi bližšího poznání společnosti univerzitních mistrů prostřednictvím soupisů jejich knihoven. Jako př́íklad uvádí inventár pozůstalosti mistra Jakuba Codicilla († 1576), v níž bylo zachyceno 216 svazků rukopisných i tištěných knih. Seznamuje s obsahem této knihovny.

Prof. PhDr. Jiř̀ Pešek

Fakulta humanitnich studii UK, Praha

jiripesek203@seznam.cz 